\title{
Targeted Notch1 inhibition with a Notch1 antibody, OMP-A2G1, decreases tumor growth in two murine models of prostate cancer in association with differing patterns of DNA damage response gene expression
}

\author{
Aqila A. Ahmed ${ }^{1}$ | Tyler Robinson ${ }^{1}$ | Monica Palande ${ }^{1}$ | June Escara-Wilke ${ }^{1}$ \\ Jinlu Dai ${ }^{1}$ Evan T. Keller ${ }^{1,2}$
}

${ }^{1}$ Department of Urology, University of Michigan, Ann Arbor, Michigan

${ }^{2}$ Biointerfaces Institute, University of Michigan, Ann Arbor, Michigan

\section{Correspondence}

Evan T. Keller, Department of Urology, University of Michigan, NCRC Building 14, Room 1162800 Plymouth Road, Ann Arbor, MI 48109-2800.

Email: etkeller@umich.edu

Funding information NIH, Grant/Award Numbers: CA093900, R01 CA093900

\begin{abstract}
Notch plays a protumorigenic role in many cancers including prostate cancer (PCa). Global notch inhibition of multiple Notch family members using $\gamma$-secretase inhibitors has shown efficacy in suppressing PCa growth in murine models. However, global Notch inhibition is associated with marked toxicity due to the widespread function of many different Notch family members in normal cell physiology. Accordingly, in the current study, we explored if specific inhibition of Notch1 would effectively inhibit PCa growth in a murine model. The androgen-dependent $\mathrm{VCaP}$ and androgen-independent DU145 cell lines were injected subcutaneously into mice. The mice were treated with either control antibody 1B7.11, anti-Notch1 antibody (OMP-A2G1), docetaxel or the combination of OMP-A2G1 and docetaxel. Tumor growth was measured using calipers. At the end of the study, tumors were assessed for proliferative response, apoptotic response, Notch target gene expression, and DNA damage response (DDR) expression. OMP-A2G1 alone inhibited tumor growth of both PCa cell lines to a greater extent than docetaxel alone. There was no additive or synergistic effect of OMP-A2G1 and docetaxel. The primary toxicity was weight loss that was controlled with dietary supplementation. Proliferation and apoptosis were affected differentially in the two cell lines. OMP-A2G1 increased expression of the DDR gene GADD45 $\alpha$ in $\mathrm{VCaP}$ cells but downregulated GADD45 $\alpha$ in Du145 cells. Taken together, these data show that Notch1 inhibition decreases PCa xenograft growth but does so through different mechanisms in the androgen-dependent $\mathrm{VCaP}$ cell line vs the androgenindependent DU145 cell line. These results provide a rationale for further exploration of targeted Notch inhibition for therapy of PCa.
\end{abstract}

K E Y W O R D S

DNA damage response, hormone sensitivity, Notch signaling, Notch1, prostate cancer 


\section{INTRODUCTION}

Notch is an evolutionarily conserved pathway with roles in various cellular processes including proliferation, apoptosis, migration, growth, and differentiation. Notch signaling is mediated by four receptors (Notch 1-4) and five ligands (delta-like 1, 3, and $4 \&$ jagged 1 and 2). ${ }^{1}$ The transmembrane surface receptors (Notch1-4) on one cell interact with membrane-bound ligands expressed on the surface of an adjacent cell. Through a series of proteolytic events mediated by $\gamma$-secretase, the Notch intracellular domain (NICD) is released from the interior cell membrane into the cytoplasm and translocates into the nucleus, where it participates in a core transcriptional complex that turns on target genes. Notch signaling is deregulated during initiation and progression of prostate cancer (PCa). ${ }^{2}$

$\mathrm{PCa}$ is the most common cancer of men. Proliferation, maintenance, and function of prostate cells is regulated by a number of signaling pathways, including Notch, DNA damage response (DDR) and the androgen receptor (AR). In both clinical samples and in cell culture, multiplicity in AR, Notch, and DDR activity has been shown to impact PCa biology and therapy response. It has been demonstrated that the androgen-signaling pathway and Notch pathway can regulate each other in $\mathrm{PCa}$. Yu et $\mathrm{al}^{3}$ showed that in PCa patients, jagged 1 expression correlates with cancer progression in a process mediated through AR overexpression. Another study reported that Heyl, a transcription factor activated by Notch1, repressed AR-dependent transcriptional activity. ${ }^{4}$ However, this repression is absent in $\mathrm{PCa}$ tumors, due to exclusion of Heyl from the nucleus in tumor cells, suggesting that progression to aggressive ARindependent $\mathrm{PCa}$ could in part be due to nuclear exclusion of repressors. ${ }^{4}$ Therefore, further understanding the interplay between AR and Notch signaling in PCa may uncover additional mechanisms through which Notch contributes to PCa progression.

Previously, we explored the impact of global Notch inhibition using a $\gamma$-secretase inhibitor (GSI) on two PCa hormone insensitive cell lines (PC3 and Du145). The GSI induced a combination of decreased proliferation, induced apoptosis, and blocked tumor angiogenesis. ${ }^{5}$ There are several drawbacks to use of global Notch inhibition including marked toxicity and targeting multiple Notch receptors as opposed to those specifically shown to be oncogenic. This is of particular therapeutic relevance as Notch can function either as a tumor-suppressive or an oncogenic mediator in PCa. ${ }^{6}$ Hence, targeting specific Notch pathway molecules that have protumor-promoting activity, while sparing Notch pathway molecules that have antitumor activity may provide a therapeutic benefit. Therefore, in this study, we investigated the therapeutic efficacy of anti-Notch1 monoclonal antibody on both hormone-independent and -dependent $\mathrm{PCa}$ xenografts.

\section{MATERIALS AND METHODS}

\section{1 $\quad$ Antibodies}

OMP-A2G1 (anti-Notch1) and the control antibody 1B7.11 (against dinitrophenol) monoclonal antibody were provided by OncoMed Pharmaceuticals (Redwood City, CA).

\subsection{Cell lines and cell culture condition}

Human prostate cancer cell lines Du145 was obtained from the American Type Culture Collection (Rockville, MD) and cultured in Rosewell Park Memorial Institute 1640 medium (Invitrogen Co, Carlsbad, CA) supplemented with $10 \%$ fetal bovine serum (FBS) and $1 \%$ penicillinstreptomycin (Life Technologies, Carlsbad, CA). ${ }^{7,8}$ VCaP cells (kindly provided by Dr. Kenneth Pienta, University of Michigan, Ann Arbor, MI) were maintained in Dulbecco's modified Eagle's medium (Life Technologies, Inc). ${ }^{9}$ with $10 \%$ FBS and $1 \%$ penicillin-streptomycin (Life Technologies). All cultures were maintained at $37^{\circ} \mathrm{C}, 5 \%$ $\mathrm{CO}_{2}$, and $100 \%$ humidity. Cell identification is completed semiannually using polymerase chain reaction (PCR) for short tandem repeats.

\subsection{Cell growth assay}

Du145 and VCaP cells were grown in 96-well plates. Cells were then treated with either GSI(R04), OMP-A2G1 (0.1, 1,10 , and $100 \mu \mathrm{g} / \mathrm{mL}$ ) or vehicle for 72 hours. Cell proliferation reagent WST-1 was added and incubated at $37^{\circ} \mathrm{C}$ and $5 \% \mathrm{CO}_{2}$ for 4 hours. Absorbance was then measured at $440 \mathrm{~nm}$ with a SpectraMax M5 plate reader. Data are presented as mean \pm SD from triplicate determinations.

\section{4 $\mid$ Animal studies}

Six to seven-week-old, male CB17/Icr-Prkdcscid/IcrIcoCrl (SCID) mice (Charles River, Wilmington, MA) were housed under pathogen-free conditions. All experimental protocols were approved by the University of Michigan Animal Care and Use Committee. For subcutaneous injection, $1 \times 10^{6}$ of Du145 cells and $3 \times 10^{6}$ of $\mathrm{VCaP}$ cells were injected subcutaneously into the flank. When cohorts of tumors reached 100 to $200 \mathrm{~mm}^{3}$, mice were randomly assigned to control and three treatment groups (13-15 mice per group): 
(1) Control antibody 1B7.11 (against dinitrophenol; (2) OMP-A2G1 ( $5 \mathrm{mg} / \mathrm{kg}$ weekly); (3) control antibody plus docetaxel (5 mg/kg weekly); (4) OMP-A2G1 plus docetaxel (same dosage as above). Tumor size and body weight were measured twice a week. Tumor volume was calculated following the formula: tumor volume $V=a \times b^{2} \times 0.52$, where $a$ is the length of the tumor and $b$ is the width of the tumor. $^{5}$

\subsection{Western blot analysis}

Du145 and VCaP subcutaneous xenografts were harvested after treatment and stored at $-80^{\circ} \mathrm{C}$. Tumor tissues were homogenized in ice-cold radioimmunoprecipitation assay lysis buffer (Millipore, Billerica, MA) containing protease inhibitors and phosphatase inhibitors. The protein concentration of tumor extracts was determined using the Peirce BCA Protein Assay Kit (ThermoFisher, Waltham, MA). Target protein expression was analyzed using Western blot analysis, with $\beta$-tubulin/glyceraldehyde 3-phosphate dehydrogenase (GAPDH) used as loading controls. Notch1, NICD, GADD45 $\alpha$, and p53 were obtained from Cell Signaling Technology Company (Beverly, MA); $\beta$-tubulin and GAPDH were obtained from Sigma-Aldrich (St Louis, MO). The antibodies were diluted as recommended by the manufacturers.

\subsection{Quantitative real-time PCR}

Total RNA was extracted from Du145 and VCaP subcutaneous xenografts using RNeasy Mini Kit (Qiagen, Valencia, CA) according to the manufacturer's protocol. Using the Superscript III first-strand synthesis system (Invitrogen, Inc), messenger RNA (mRNA) was reversetranscribed into complementary DNA (cDNA). SYBR green (Qiagen) was used to amplify cDNA using quantitative realtime reverse transcriptase polymerase chain reaction (qRTPCR). Relative quantification was done using the $\Delta \Delta C_{\mathrm{t}}$ method, normalizing to GAPDH mRNA.

\subsection{Immunohistochemistry}

Subcutaneous tumors were fixed in $10 \%$ formalin. Five-micron $(5 \mu \mathrm{M})$ sections were used for hematoxylineosin and immunohistochemistry. Ki67 (1:500; Santa Cruz Biotechnology, Dallas, TX) and cleaved caspase-3 (1:300; Cell Signaling Technology, Danvers, MA) were stained as per the manufacturer's protocols. Sections were examined for positive staining and quantified as previously described. ${ }^{5}$ Representative fields were photographed under $\times 40$ magnification.

\subsection{Statistical analysis}

Numerical data are expressed as mean \pm SD. Statistical analysis was performed by one-way analysis of variance for multiple comparisons and the Student $t$ test for an independent analysis. Differences with $P<0.05$ were determined as statistically significant.

\section{\begin{tabular}{l|l}
3 & RESULTS
\end{tabular}}

\section{1 | Anti-Notch1 antibody decreases proliferation of hormone-sensitive prostate cancer cells}

We first explored baseline gene expression levels of the Notch ligands, receptors, and Notch pathway activation in several prostate cancer cells. Initially, RT-PCR analysis was performed revealing a varied expression of among the PCa cell lines. Downstream target genes of the Notch pathway were higher in VCaP compared to the other two cell lines (Figure 1A). In terms of protein expression, Notch1 was highest in DU145 and lowest in VCaP; whereas, it was expressed at various levels among the other prostate cancer cell lines (Figure 1B). These results confirm previous work that VCaP cells express Notch1 protein $^{10}$ as did DU145 cells. ${ }^{11-13}$ We next explored if Notch1 inhibition impacted the growth of the PCa cells lines. First, we treated cells with the GSI R04 for 72 hours to determine the impact of global Notch inhibition on these cells. R04 inhibited overall cell growth of both cell lines in a dose-responsive fashion (Figure 1C). We next determined the impact of specific inhibition of Notch1 on prostate cancer cell growth. Accordingly, we cultured the prostate cancer cells with increasing doses of anti-Notch1 monoclonal antibody OMP-A2G1 for 72 hours. OMPA2G1 reduced cell growth of both cell lines in a doseresponsive fashion with more of an inhibitory impact VCaP compared to DU145 (Figure 1D). The response in VCaP with low Notch1 expression compared to Du145 supports the efficacy of OMP-A2G1. These results indicate that Notch1 can promote the growth of both androgen-sensitive and insensitive PCa cells.

\section{2 | Notch1 inhibits the growth of both hormone-dependent and -independent xenografts}

To investigate the potential therapeutic efficacy of OMP-A2G1 in vivo, we subcutaneously injected VCaP and Du145 into mice and allowed tumors to become established to a volume range of 100 to $200 \mathrm{~mm}^{3}$. At this point, mice were randomized into four treatment groups consisting of control antibody 1B7.11 (against 
(A)

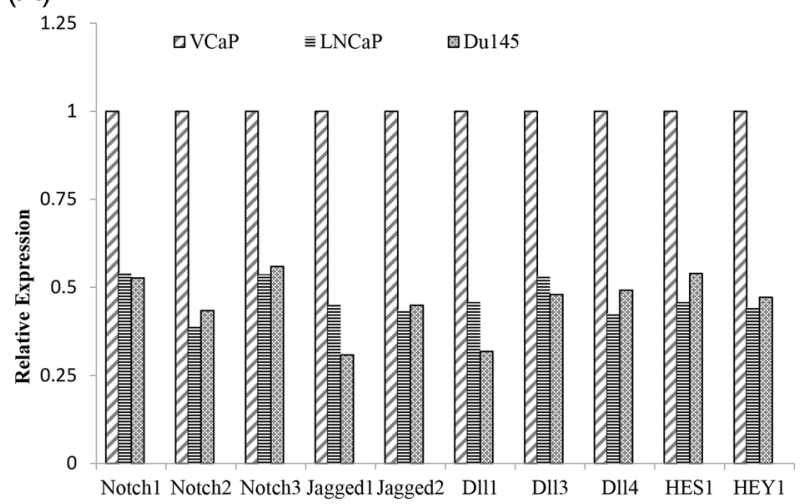

(B)

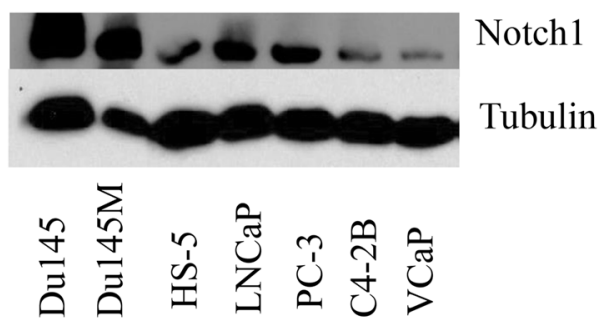

(C)

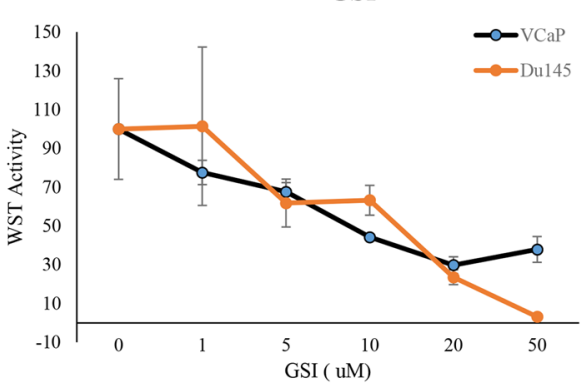

(D)

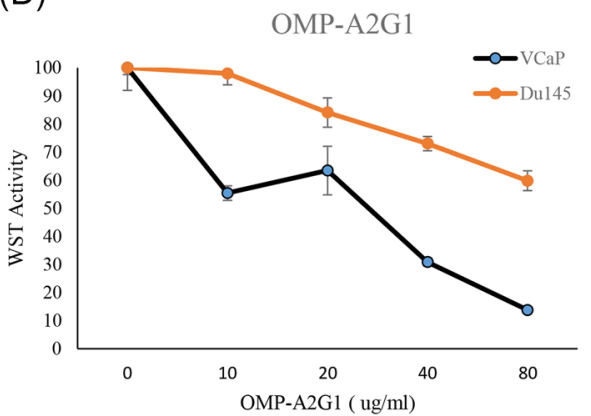

F I G U RE 1 Notch signaling evaluation in hormone-dependent and -independent prostate cancer cell lines. A, To measure mRNA, total RNA was extracted from three different prostate cancer cell lines (LNCaP, DU145, and VCaP) and $1 \mu \mathrm{g}$ of total RNA was reverse-transcribed and subjected to qRT-PCR for the indicated target gene and normalized to GAPDH and then reported relative to VCaP. B, To measure protein, indicated cell lines were subjected to immunoblot for anti-Notch1 antibody. $\beta$-Tubulin was used as a loading control. $\mathrm{C}, 3 \times 10^{3}$ DU145 and VCaP cells were treated with GSI R04 for 72 hours. Cell viability was measured using resazurin cell viability assays. D, $3 \times 10^{3}$ DU145 and VCaP cells were treated with OMP-A2G1 $(0-80 \mu \mathrm{g} / \mathrm{mL}) 72$ hours. Cell viability was measured using resazurin cell viability assays. GAPDH, glyceraldehyde 3-phosphate dehydrogenase; mRNA, messenger RNA; qRT-PCR, quantitative real-time reverse transcriptase polymerase chain reaction. ${ }^{*} P<0.05$ and ${ }^{* *} P<0.01$

dinitrophenol), control antibody plus docetaxel, OMPA2G1 alone, or OMP-A2G1 plus docetaxel and treated as indicated in Figure 2A over a 6-week period. Docetaxel inhibited the growth of $\mathrm{VCaP}$ xenografts compared to the control group (Figure 2B). OMP-A2G1 inhibited the growth of $\mathrm{VCaP}$ xenografts to a greater extent than docetaxel alone did. The combination of docetaxel and OMP-A2G1 had an inhibitory effect similar to that of OMP-A2G1 alone indicating that there was no additive or synergistic effect for these two compounds. These growth effects are reflected in the final tumor weights of all four groups of $\mathrm{VCaP}$ xenografts (Figure 2B). OMP-A2G1 had a similar impact on the Du145 xenografts with OMP-A2G1 inhibiting tumor growth more than docetaxel (Figure 2C). Taken together, these results indicate that OMP-A2G1 has a strong antitumor effect; however, there was no additive or synergistic activity between docetaxel and OMP-A2G1. OMP-A2G1 primary toxicity was weight loss, which was controlled with dietary supplementation.

\subsection{Anti-Notch1 exerts mixed effects}

To determine if Notch1 inhibition mediates its antitumor effect through modulating proliferation and/or apoptosis in vivo, we assessed the expression of Ki67 (proliferation marker) and cleaved caspase-3 (apoptosis marker) in the VCaP and DU145 xenografts. None of the solitary or combination treatments had an impact on the number of cells expressing Ki67 in the VCaP xenografts (Figure 3A). In contrast, OMP-A2G1 and docetaxel alone increased the number of cleaved caspase- 3 positive cells; however, the combination of OMP-A2G1 and docetaxel had no impact on the number of cleaved caspase- 3 positive cells. In $\mathrm{VCaP}$ xenograft, OMP-A2G1 and docetaxel alone had no impact on the number of cells expressing Ki67; although, the combination of both decreased the proportion of Ki67 positive cells in the Du145 xenografts (Figure 3B). Furthermore, none of the individual or combination treatments had an impact on the number of cells expressing caspase-3. 

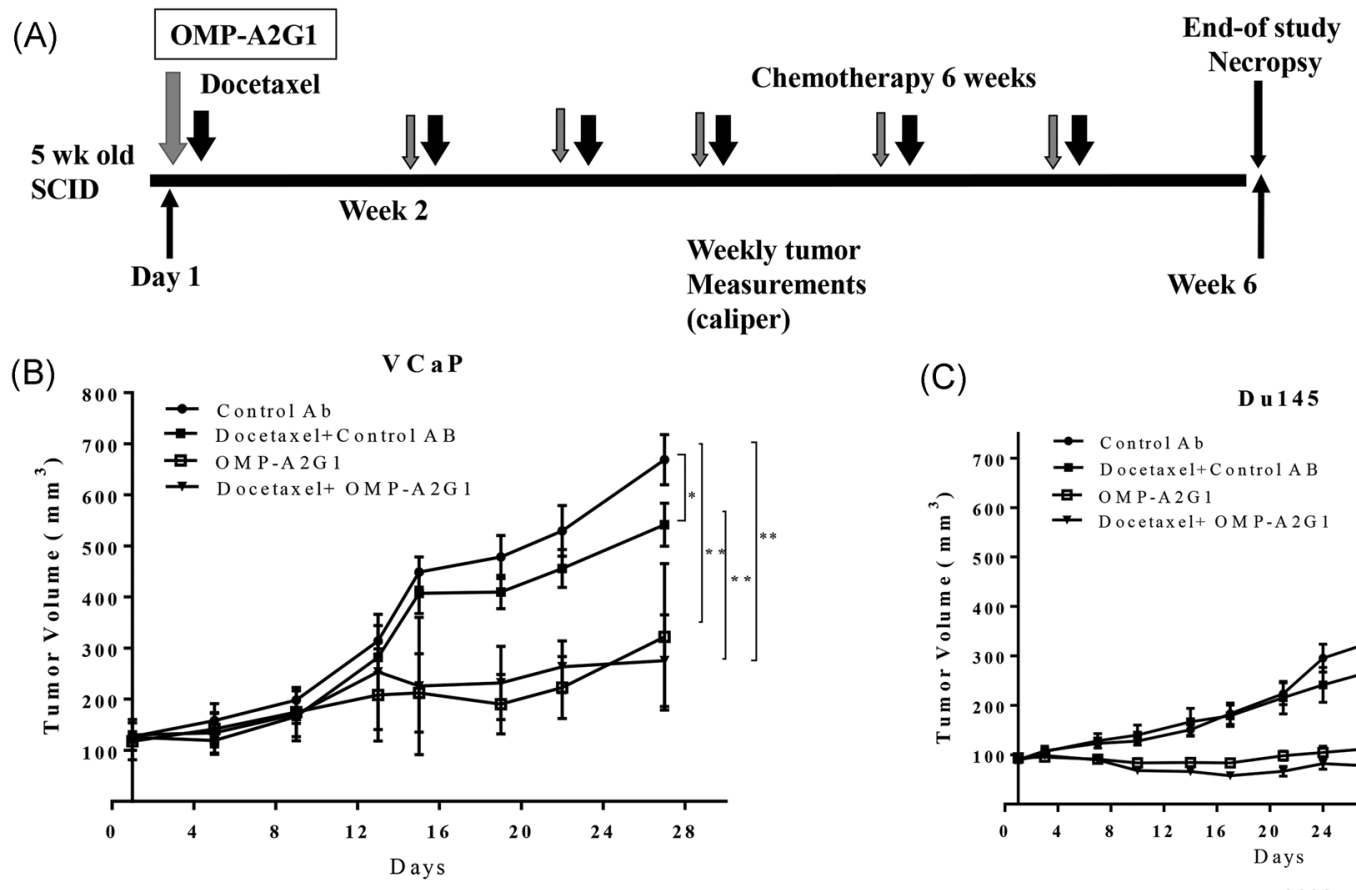

(C)
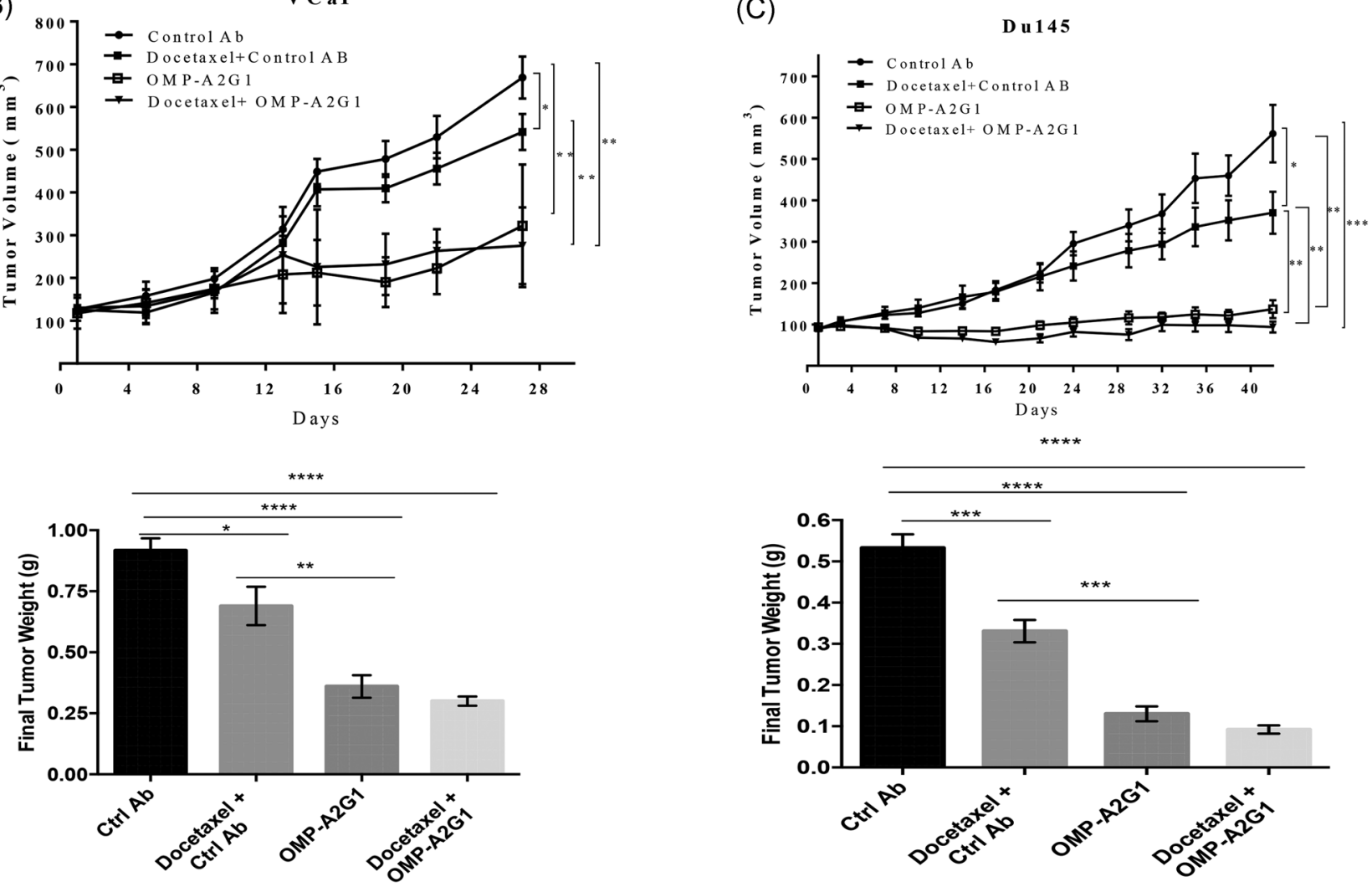

FIG URE 2 OMP-A2G1 had a significant antitumor effect in VCaP and Du145 xenografts. A, General scheme for OMP-A2G1 and docetaxel treatment. B,C, Du145 $\left(1 \times 10^{6}\right)$ and VCaP $\left(3 \times 10^{6}\right)$ were injected subcutaneously into mice. When cohorts of tumors reached 100 to $200 \mathrm{~mm}^{2}$ mice were divided into four treatment groups consisting of control antibody $1 \mathrm{~B} 7.11$ (against dinitrophenol), control antibody plus docetaxel, OMP-A2G1 alone, or OMP-A2G1 plus docetaxel and treated with as described in Section $2(N=12-14$ mice per group). D, At the end of the study, tumors were harvested and weighted, mean weights were then graphed. NS, no significant difference. ${ }^{*} P<0.05$,

** $P<0.001$, and $* * * P<0.0001$

\section{4 | The response of Notch target genes to Notch1 varies in xenografts}

To determine the effect of Notch1 inhibition on Notch pathway-related genes in the tumor tissues, we subjected the VCaP and Du145 xenografts to Western blot analysis. In response to OMP-A2G1 antibody alone, $\mathrm{VCaP}$ xenografts did not show a decrease of NICD protein expression (Figure 4A); whereas, the Notch target, Hes1 mRNA expression was significantly decreased (Figure 4C). In contrast in DU145, the OMP-A2G1 antibody alone decreased NICD protein expression, but not Hes1 mRNA expression (Figure 4B,D). The combination of docetaxel and OMP-A2G1 did significantly decrease Hes1 mRNA expression in Du145 xenografts (Figure 4D). We looked at additional Notch1 targets such as Hes6, Hey1, and Notch3. In VCaP xenografts, OMPA2G1 treatment led to a downregulation trend in these transcripts; whereas, there was no impact on them in DU145 cells (data not shown). Taken together, these results indicate that OMP-A2G1 antibody impacts Notch1-mediated signaling in these PCa cells and this might be by different mechanisms. 
(A) $\mathrm{VCaP}$

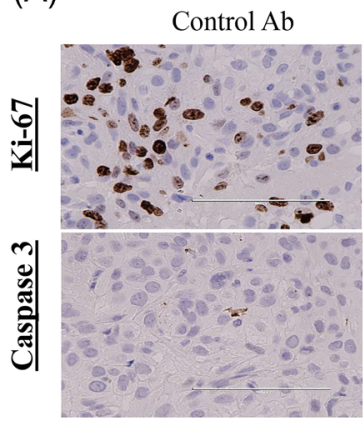

Control Ab+DOX
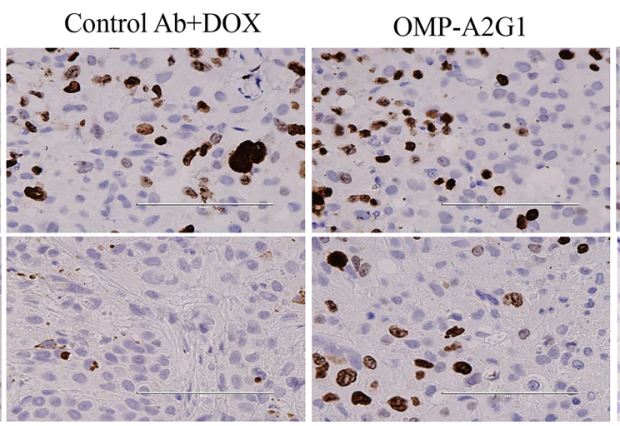

(B) Du145

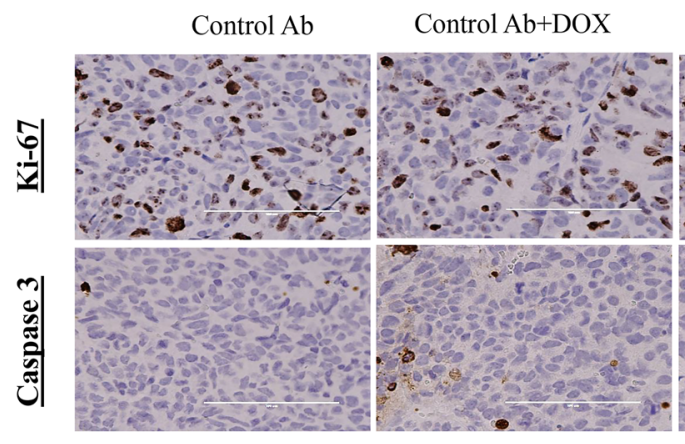

OMP-A2G1

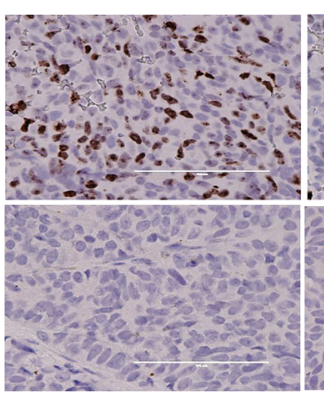

OMP-A2G1 +DOX

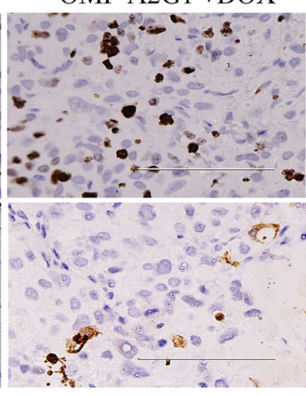

OMP-A2G1 +DOX

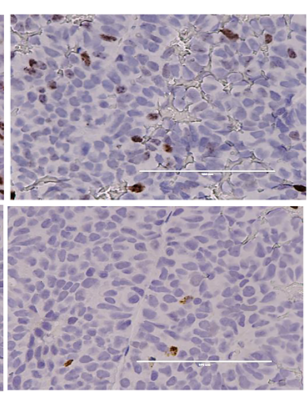

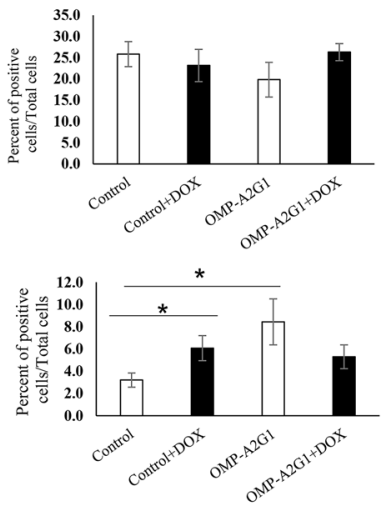

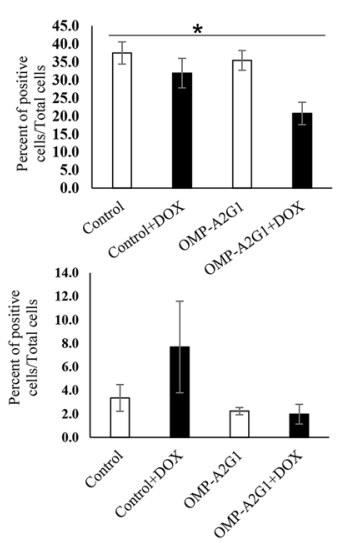

F I G URE 3 : OMP-A2G1 induces apoptosis alone and in combination with docetaxel in DU145 and suppresses tumor cell proliferation in VCaP. A,B, At the end of the study, tumors were harvested from the mice as described in methods. A portion of the DU145 and VCaP tumor xenografts $(n=5)$ were subjected to immunohistochemistry for proliferation (anti-Ki67) and apoptosis (anticleaved caspase-3). Left: Representative photomicrographs of Ki67 and cleaved caspase-3 stained tumor sections ( $\times 40$ magnification). Right: Quantitation of Ki67 and cleaved caspase- 3 positive percentage. ${ }^{*} P<0.05$ and ${ }^{* *} P<0.01$

\subsection{Intrinsic PCa cell line properties influence response to Notch1 inhibition}

We next explored why the two different PCa cell lines demonstrated differential responses to OMP-A2G1 therapy. Notch signaling is involved in a wide range of cellular processes and any one of these can be altered depending on the state of the cell. We focused on the underlying differences between our models. $\mathrm{VCaP}$ is $\mathrm{AR}$ and ERG positive with a functional mutated p53 protein while DU145 is both AR and ERG negative with a nonfunctional p53 possible. ${ }^{15}$ Mohamed et al ${ }^{14}$ reported synergy between Notch and AR inhibition in VCaP but not DU145. VCaP cells had decreased cell growth, cell survival, and enhanced apoptosis in response to Notch and the AR inhibitor bicalutamide while no change was observed in AR-negative DU145 cells. ${ }^{14}$ DU145 showed no change in protein expression after treatment with Notch and AR inhibitors. Another inherent difference is the p53 status of $\mathrm{VCaP}$ and DU145. DU145 harbors mutant Leu-223 and Phe-274 that renders p53 nonfunctional, while VCaP (mutant p53, Trp-248) has been shown to have a functional p53 protein. ${ }^{15,16}$ Carroll et $\mathrm{al}^{15}$ showed that DDR in PCa cell lines was dependent on p53 status and that alteration of this pathway is vital in the survival of advanced PCa cells during exposure to anticancer therapies, specifically agents that induce DNA damage. Since DDR is dependent on p53 functional status, which is different between VCaP and DU145 cells, we evaluated the effect of OMP-A2G1 on DDR. To perform this, we utilized an array consisting of genes involved in Ataxia telangiectasia-mutated and RAD3-related (ATM/ATR) signaling, DDR, apoptosis, and cell cycle. Equal amounts of RNA from each xenograft was characterized for the same 84 genes involved in DNA damage signaling pathways. $\mathrm{VCaP}$ tumors treated with OMP-A2G1 had dysregulation of genes involved in cell cycle and apoptosis (Figure 5A). In contrast, DU145 did not show any dysregulated genes in common with $\mathrm{VCaP}$ but had dysregulation in several DDR genes (Figure 5B). Although the dysregulated genes were different among VCaP and DU145 cells, they have a p53 pathway in 
(A)

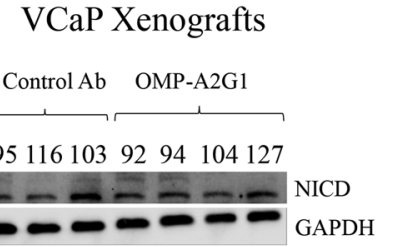

(C)

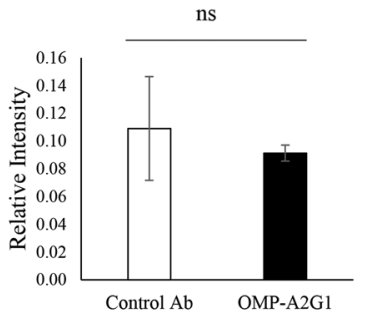

(B)

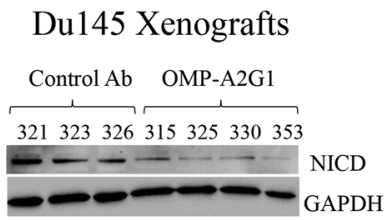

(D)

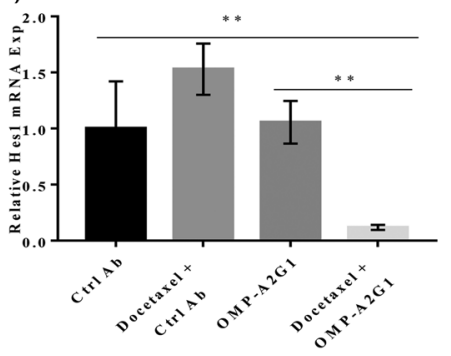

F IG U RE 4 Differential Notch signaling response to OMP-A2G1 treatment. A,B, Total protein was isolated from OMP-A2G1 and control-treated DU145 and VCaP and subject to immunoblot for NICD1 as described in Figure 1. GAPDH was used as a loading control. Bands were measured using densitometry and values first normalized to respective GAPDH bands. C,D, To measure mRNA, total RNA was extracted from OMP-A2G1 and control-treated DU145 and VCaP. Total RNA $(1 \mu \mathrm{g})$ was subjected to qRT-PCR using primers for Hes1 and GAPDH (as a loading control). GAPDH, glyceraldehyde 3-phosphate dehydrogenase; mRNA, messenger RNA; NICD1, Notch intracellular domain; qRT-PCR, quantitative real-time reverse transcriptase polymerase chain reaction. ${ }^{*} P<0.05$ and ${ }^{* *} P<0.01$

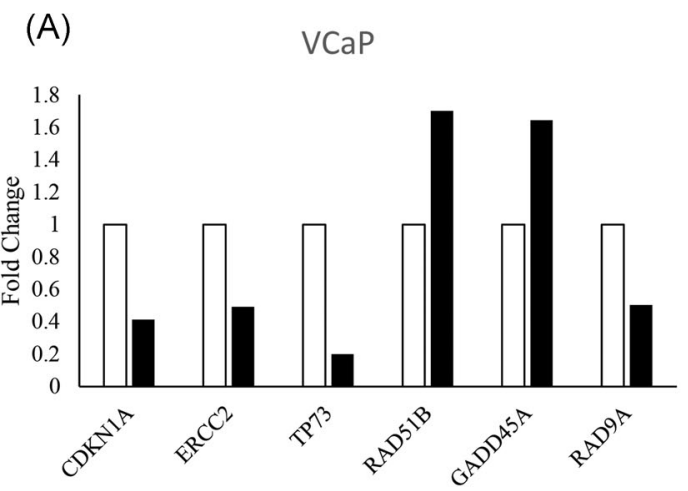

(B)

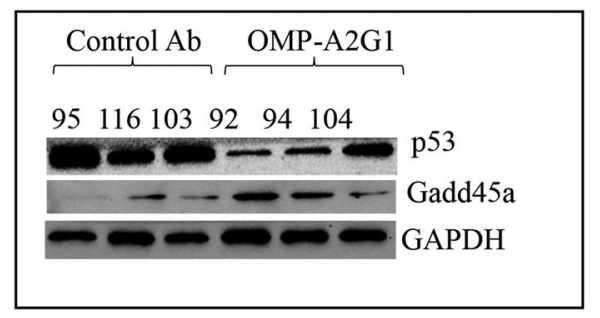

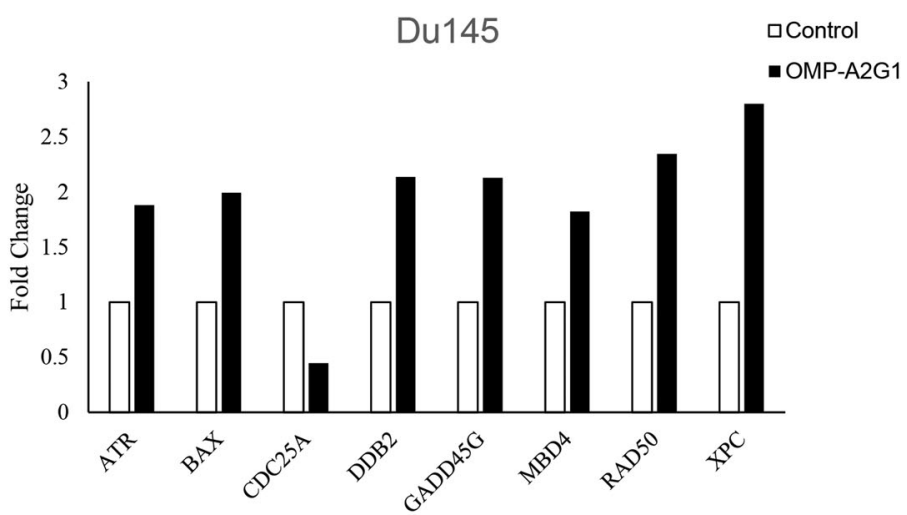

(C)

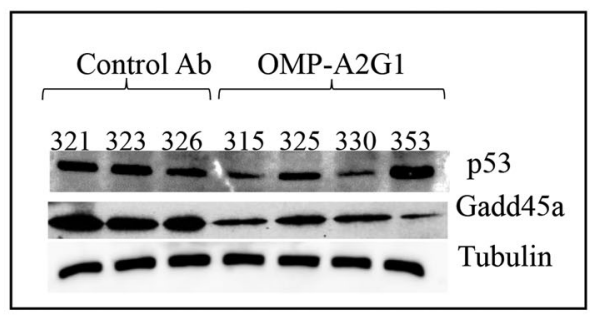

FI G U R E 5 OMP-A2G1-treated VCaP and Du145 xenografts have differential DNA damage response. A, Four hundred nanograms of total RNA was extracted from DU145 and VCaP-treated tumors and their respective control antibody-treated tumors and was reversetranscribed and subjected to qRT-PCR using the human DNA damage signaling pathway PCR array. $C_{\mathrm{t}}$ values were analyzed using the GeneGlobe Data Analysis Center, relative gene expression between of top genes in each xenograft was then plotted. B,C, Total protein was isolated from OMP-A2G1 and control-treated DU145 and VCaP and subject to immunoblot for p53 and GADD45 $\alpha$ as described in Figure 1. GAPDH and $\beta$-tubulin were used as a loading control. GAPDH, glyceraldehyde 3-phosphate dehydrogenase; qRT-PCR, quantitative real-time reverse transcriptase polymerase chain reaction 
common. Notch signaling has been shown to regulate p53 expression via both Hey1 and Hes $1^{17}$ as well as DDR. ${ }^{18}$ Accordingly, we explored for an impact of Notch1 inhibition on p53 and Gadd45 $\alpha$, which is a downstream target of p53 in DDR. In VCaP xenografts, OMP-A2G1 downregulated p53 expression (Figure 5B). This was associated with an increase in downstream target Gadd45 $\alpha$. In contrast, in DU145 cells, which lack a functional p53, OMP-A2G1 had no impact on p53 expression but did decrease Gadd45 $\alpha$ (Figure 5C). These results suggest that $\mathrm{p} 53$ function could contribute to a differential impact of Notch1 inhibition in $\mathrm{PCa}$ cells.

\section{DISCUSSION}

Notch signaling is multifaceted and is known to associate with multiple processes including p53 and androgen-signaling pathways. In our study, we evaluated the response to OMP-A2G1 in androgen-dependent and independent cells. OMP-A2G1 reduced the growth of both DU145 and VCaP xenografts. The overall impact of OMP-A2G1 on cellular mechanisms (ie, decreased proliferation or increased apoptosis) accounting for the decreased tumor growth appeared different between the DU145 and VCaP xenografts. Specifically, while $\mathrm{VCaP}$ showed a significant upregulation in cleaved caspase-3, DU145 xenografts showed no difference between treatment groups. Both cell lines have inherent differences not only in AR but ERG and p53 expression, which may account for the differential response. Canonical and noncanonical Notch are known to crosstalk with AR and p53. ${ }^{16}$ Considering all of these, we took a closer look at p53 and DDR and showed differences in response between the xenografts exposed to OMP-A2G1. Gadd45 $\alpha$, a proapoptotic and p53-dependent DDR protein was upregulated in $\mathrm{VCaP}$ xenografts in response to OMP-A2G1. Recent work has attributed GADD45 $\alpha$ upregulation to $\mathrm{VCaP}$ expression of fusion protein TMPRSS2-ERG. ${ }^{22}$ DU145 cells express mutant nonfunctional p53 that causes a defective $\mathrm{G}_{1}$ checkpoint $^{16}$ which can account for the lack of Gadd45 $\alpha$ upregulation upon OMP-A2G1 treatment. These results are supportive of the possibility that DNA damage-induced p53 contributes to the increased apoptosis seen in VCaP when Notch1 is inhibited. Loss of p53 function can relieve apoptosis and lead to growth advantage. ${ }^{23}$ It's been reported in multiple cancers that p53 loss is synergistic with Notch1 expression.

Given that Notch1 has a spectrum of activities, our data suggest that Notch1 inhibition can impede $\mathrm{PCa}$ growth through various mechanisms such as DDR, AR, and p53 signaling. Recent work on Notch signaling and DDR shows that Notch1 can displace key DDR proteins and alter the response to damage. ${ }^{18}$ The crosstalk between AR and Notch1 is also crucial in the response to Notch inhibition. Downstream targets of Notch signaling Hey1, Hey2, and HeyL were shown to repress AR signaling. ${ }^{4,29}$ Our data show an upregulation of AR in anti-Notch1 treated VCaP tumors (data not shown). Mohamed et al, ${ }^{14}$ showed that Notch1 and Notch2 have ERG binding sites upstream of promoters in the TMPRSS2-ERG positive VCaP but not DU145. They also show that dual AR and Notch inhibition repressed proliferation and increased apoptosis in $\mathrm{VCaP}$ but not DU145. Kron et $\mathrm{al}^{30}$ recently showed that TMPRSS2ERG fusion activates Notch signaling in primary PCa. Furthermore, Notch inhibition was shown to overcome resistance to androgen deprivation in $\mathrm{PCa}$ cells, strengthening the link between Notch and AR signaling. ${ }^{31}$

VCaP AR expression may explain the lack of difference in proliferation of OMP-A2G1-treated tumors. Another possibility is the ERG expression present in $\mathrm{VCaP}$ but not DU145. Mohamed et $\mathrm{al}^{14}$ showed that AR and Notch inhibition delivered a significant increase in apoptotic cells and a decrease in cell growth and cell survival in VCaP, while DU145 showed no difference.

In summary, our study demonstrates that specific Notch1 inhibition reduces PCa xenograft growth. The data indicates the antitumor effects may be associated with DDR and AR status. These results suggest that further exploration of targeted Notch inhibition in combination with DNAdamaging agents and AR inhibitors may lead to promising $\mathrm{PCa}$ therapies.

\section{ACKNOWLEDGMENTS}

We thank Tim Hoey and OncoMed for provision of OMPA2G1 and 1B7.11 antibodies. This study was supported in part by NIH CA093900.

\section{CONFLICT OF INTERESTS}

The authors declare that there are no conflict of interests.

\section{REFERENCES}

1. Su Q, Xin L. Notch signaling in prostate cancer: refining a therapeutic opportunity. Histol Histopathol. 2016;31(2):149-157. https://doi.org/10.14670/HH-11-685

2. Bertrand FE, McCubrey JA, Angus CW, Nutter JM, Sigounas G. NOTCH and PTEN in prostate cancer. Adv Biol Regul. 2014;56:51-65. https://doi.org/10.1016/j.jbior.2014.05.002 
3. Yu Y, Zhang Y, Guan W, et al. Androgen receptor promotes the oncogenic function of overexpressed Jagged1 in prostate cancer by enhancing cyclin B1 expression via Akt phosphorylation. Mol Cancer Res. 2014;12(6):830-842. https://doi.org/10.1158/ 1541-7786.mcr-13-0545

4. Belandia B, Powell SM, Garcia-Pedrero JM, Walker MM, Bevan CL, Parker MG. Hey1, a mediator of Notch signaling, is an androgen receptor corepressor. Mol Cell Biol. 2005;25(4):14251436. https://doi.org/10.1128/MCB.25.4.1425-1436.2005

5. Cui D, Dai J, Keller JM, Mizokami A, Xia S, Keller ET. Notch pathway inhibition using PF-03084014, a gamma-secretase inhibitor (GSI), enhances the antitumor effect of docetaxel in prostate cancer. Clin Cancer Res. 2015;21(20):4619-4629. https://doi.org/10.1158/1078-0432.CCR-15-0242

6. Carvalho FL, Simons BW, Eberhart CG, Berman DM. Notch signaling in prostate cancer: a moving target. Prostate. 2014;74(9):933-945. https://doi.org/10.1002/pros.22811

7. Sampson N, Neuwirt H, Puhr M, Klocker H, Eder IE. In vitro model systems to study androgen receptor signaling in prostate cancer. Endocr Relat Cancer. 2013;20(2):R49-R64. https://doi. org/10.1530/erc-12-0401

8. Yu S-Q, Lai K-P, Xia S-J, Chang H-C, Chang C, Yeh S. The diverse and contrasting effects of using human prostate cancer cell lines to study androgen receptor roles in prostate cancer. Asian J Androl. 2009;11(1):39-48. https://doi.org/10.1038/aja. 2008.44

9. Korenchuk S, Lehr JE, L MC, et al. VCaP, a cell-based model system of human prostate cancer. In Vivo. 2001;15(2):163-168.

10. Litvinov Ivan V, Vander Griend Donald J, Xu Yi, Antony Lizamma, Dalrymple Susan L, Isaacs John T. Low-Calcium Serum-Free Defined Medium Selects for Growth of Normal Prostatic Epithelial Stem Cells. Cancer Research. 2006;66(17):8598-8607. https://doi.org/10.1158/0008-5472.can06-1228

11. Shou J, Ross S, Koeppen H, de Sauvage FJ, Gao WQ. Dynamics of notch expression during murine prostate development and tumorigenesis. Cancer Res. 2001;61(19):7291-7297.

12. Zayzafoon Majd, Abdulkadir Sarki A, McDonald Jay M. Notch Signaling and ERK Activation Are Important for the Osteomimetic Properties of Prostate Cancer Bone Metastatic Cell Lines. Journal of Biological Chemistry. 2003;279(5):3662-3670. https:// doi.org/10.1074/jbc.m308158200

13. Patrawala Lubna, Calhoun Tammy, Schneider-Broussard Robin, Zhou Jianjun, Claypool Kent, Tang Dean G. Side Population Is Enriched in Tumorigenic, Stem-Like Cancer Cells, whereas ABCG2+and ABCG2-Cancer Cells Are Similarly Tumorigenic. Cancer Research. 2005;65(14):6207-6219. https://doi.org/10.1158/0008-5472.can-05-0592

14. Mohamed AA, Tan SH, Xavier CP, et al. Synergistic activity with NOTCH inhibition and androgen ablation in ERG-positive prostate cancer cells. Mol Cancer Res. 2017;15(10):1308-1317. https://doi.org/10.1158/1541-7786.MCR-17-0058

15. Carroll AG, Voeller HJ, Sugars L, Gelmann EP. p53 oncogene mutations in three human prostate cancer cell lines. Prostate. 1993;23(2):123-134.

16. Chappell WH, Lehmann BD, Terrian DM, Abrams SL, Steelman LS, McCubrey JA. p53 expression controls prostate cancer sensitivity to chemotherapy and the MDM2 inhibitor Nutlin-3. Cell Cycle. 2012;11(24):4579-4588. https://doi.org/10.4161/cc. 22852

17. Huang Q, Raya A, DeJesus P, et al. Identification of p53 regulators by genome-wide functional analysis. Proc Natl Acad Sci USA. 2004;101(10):3456-3461.

18. Vermezovic J, Adamowicz M, Santarpia L, et al. Notch is a direct negative regulator of the DNA-damage response. Nat Struct Mol Biol. 2015;22(5):417-424. https://doi.org/10.1038/ nsmb. 3013

19. Haffner MC, Aryee MJ, Toubaji A, et al. Androgen-induced TOP2B-mediated double-strand breaks and prostate cancer gene rearrangements. Nature Genet. 2010;42(8):668-675. https://doi.org/ 10.1038/ng.613

20. Leong KG, Gao WQ. The Notch pathway in prostate development and cancer. Differentiation. 2008;76(6):699-716. https:// doi.org/10.1111/j.1432-0436.2008.00288.x

21. Thompson TC, Li L. Connecting androgen receptor signaling and the DNA damage response: development of new therapies for advanced prostate cancer. $\mathrm{Mol} \mathrm{Cel}$ Oncol. 2017;4(4):e1321167-e1321167. https://doi.org/10. 1080/23723556.2017.1321167

22. Chatterjee P, Choudhary GS, Alswillah T, et al. The TMPRSS2ERG gene fusion blocks XRCC4-mediated nonhomologous end-joining repair and radiosensitizes prostate cancer cells to PARP inhibition. Mol Cancer Ther. 2015;14(8):1896-1906. https://doi.org/10.1158/1535-7163.MCT-14-0865

23. Lin RW, Ho CJ, Chen HW, et al. P53 enhances apoptosis induced by doxorubicin only under conditions of severe DNA damage. Cell Cycle. 2018;17(17):2175-2186. https://doi.org/10. 1080/15384101.2018.1520565

24. Deng G, Ma L, Meng Q, et al. Notch signaling in the prostate: critical roles during development and in the hallmarks of prostate cancer biology. J Cancer Res Clin Oncol. 2016;142(3): 531-547. https://doi.org/10.1007/s00432-015-1946-X

25. Qiu S, Deng L, Bao Y, et al. Reversal of docetaxel resistance in prostate cancer by Notch signaling inhibition. Anticancer Drugs. 2018;29(9):871-879. https://doi.org/10.1097/CAD. 0000000000000659

26. Zhang L, Sha J, Yang G, Huang X, Bo J, Huang Y. Activation of Notch pathway is linked with epithelial-mesenchymal transition in prostate cancer cells. Cell Cycle. 2017;16(10):999-1007. https://doi.org/10.1080/15384101.2017.1312237

27. Carvalho FL, Marchionni L, Gupta A, et al. HES6 promotes prostate cancer aggressiveness independently of Notch signaling. J Cell Mol Med. 2015;19(7):1624-1636. https://doi.org/10. $1111 / \mathrm{jcmm} .12537$

28. Chanrion M, Kuperstein I, Barrière $\mathrm{C}$, et al. Concomitant Notch activation and p53 deletion trigger epithelial-to-mesenchymal transition and metastasis in mouse gut. Nat Commun. 2014;5:5005. https://doi.org/10.1038/ncomms6005 https://www.nature.com/articles/ncomms6005\#supplementary-information

29. Lavery DN, Villaronga MA, Walker MM, Patel A, Belandia B, Bevan CL. Repression of androgen receptor activity by HEYL, a third member of the Hairy/enhancer-of-split-related family of Notch effectors. J Biol Chem. 2011;286(20):17796-17808. https:// doi.org/10.1074/jbc.M110.198655 
30. Kron Ken J, Murison Alexander, Zhou Stanley, Huang Vincent, Yamaguchi Takafumi N, Shiah Yu-Jia, Fraser Michael, van der Kwast Theodorus, Boutros Paul C, Bristow Robert G, Lupien Mathieu. TMPRSS2-ERG fusion co-opts master transcription factors and activates NOTCH signaling in primary prostate cancer. Nature Genetics. 2017;49(9):1336-1345. https://doi.org/10.1038/ng. 3930

31. Cui J, Wang Y, Dong B, et al. Pharmacological inhibition of the Notch pathway enhances the efficacy of androgen deprivation therapy for prostate cancer. Int J Cancer. 2018;143(3):645-656. https://doi.org/10.1002/ijc.31346
How to cite this article: Ahmed AA, Robinson T, Palande M, Escara-Wilke J, Dai J, Keller ET.

Targeted Notch1 inhibition with a Notch1 antibody, OMP-A2G1, decreases tumor growth in two murine models of prostate cancer in association with differing patterns of DNA damage response gene expression. J Cell Biochem. 2019;120: 16946-16955. https://doi.org/10.1002/jcb.28954 